\title{
Fluorofenidone attenuates paraquat-induced pulmonary fibrosis by regulating the PI3K/Akt/mTOR signaling pathway and autophagy
}

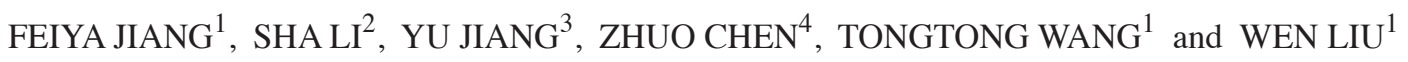 \\ ${ }^{1}$ Department of Pharmacy, The First Hospital Affiliated with Hunan Normal University, Changsha, \\ Hunan 410005; ${ }^{2}$ Department of Pharmacy, Changsha Stomatological Hospital, Changsha, Hunan 410004; \\ ${ }^{3}$ Department of Emergency, The First Hospital Affiliated with Hunan Normal University, Changsha, \\ Hunan 410005; ${ }^{4}$ Department of Pharmacy, Central South University, Changsha, Hunan 410013, P.R. China
}

Received December 25, 2020; Accepted March 15, 2021

DOI: $10.3892 / \mathrm{mmr} .2021 .12044$

\begin{abstract}
Paraquat (PQ) is a widely used herbicide that is severely toxic to humans and animals. Pulmonary fibrosis is a disorder that can result from PQ poisoning. Fluorofenidone (AKF-PD) is a novel small molecule pyridone drug with a widespread and clear anti-organ fibrosis effect; however, its mechanism of action on PQ poisoning-induced pulmonary fibrosis is not clear. The purpose of the present study was to investigate the protective effect and underlying mechanism of AKF-PD on PQ poisoning-induced pulmonary fibrosis. Human alveolar epithelial cells (HPAEpiC) and Sprague-Dawley rats were treated with AKF-PD in the presence or absence of PQ. Hematoxylin-eosin and Masson staining were used to observe the morphological changes in lung tissue. Cell Counting Kit-8 and lactate dehydrogenase assays were used to evaluate the viability of HPAEpiC cells. ELISA was used to detect inflammatory factors and the collagen content. Finally, the effects of AKF-PD on pulmonary fibrosis, as well as the underlying mechanisms, were evaluated via western blotting, reverse transcription-quantitative PCR and immunofluorescence analysis. AKF-PD effectively alleviated PQ-induced pulmonary fibrosis and reduced the expression of oxidative stress and inflammatory factors. Moreover, AKF-PD treatment effectively inhibited the PI3K/Akt/mTOR signaling pathway and upregulated autophagy. Overall, these findings suggested that AKF-PD can alleviate PQ-induced inflammation and pulmonary fibrosis by inhibiting the PI3K/Akt/mTOR signaling pathway and by upregulating autophagy.
\end{abstract}

Correspondence to: Dr Wen Liu, Department of Pharmacy, The First Hospital Affiliated with Hunan Normal University, 61 Jiefang West Road, Furong, Changsha, Hunan 410005, P.R. China

E-mail: liuwen@hunnu.edu.cn

Key words: fluorofenidone, paraquat, pulmonary fibrosis, autophagy, mTOR

\section{Introduction}

Paraquat (PQ) is a non-selective herbicide that is widely used in developing countries (1). It is toxic to humans and animals, and the lung is its main target organ (2). Severe lung injury often occurs after PQ poisoning, which is characterized by alveolar epithelial cell damage, edema, exudation and interstitial changes, as well as inflammatory cell infiltration and progressive fibrosis that can culminate in death due to respiratory failure. Pulmonary fibrosis is one of the most typical manifestations of $\mathrm{PQ}$ poisoning (3).

The mechanism of PQ-induced pulmonary fibrosis remains unclear. It is usually considered that inflammation and oxidative stress may be involved in the pathogenesis of pulmonary fibrosis (4). In the early stages of PQ-induced acute lung injury, inflammatory cells are activated and the influx of neutrophils and macrophages increases the level of proinflammatory cytokines (5). Furthermore, the production of superoxide radicals significantly increases and directly or indirectly damages alveolar epithelial cells (6). In addition, fibrogenic factors such as TGF- $\beta$, collagen and $\alpha$-smooth muscle actin ( $\alpha$-SMA) also promote the process of pulmonary fibrosis (7).

At present, the treatment guidelines for patients with $\mathrm{PQ}$ poisoning are evolving. The current treatment methods mainly include: i) Emetic induction; ii) catharsis; iii) gastric lavage; iv) hemodialysis; v) antioxidant treatment; vi) immune regulation; and vii) hemoperfusion (8). Despite these treatments, the strong toxicity of PQ poisoning and the lack of specific antidotes for it have resulted in mortality that is still $>50 \%$ (9). Therefore, the development of effective drugs for the treatment of pulmonary fibrosis is needed.

Fluorofenidone [1-(3-fluorophenyl)-5-methylpyridin-2(1H)-1; AKF-PD] is a novel, small-molecule pyridone drug with clear and extensive antifibrotic effects (10). AKF-PD inhibits fibrosis by reducing inflammation and oxidative stress, inhibiting cell proliferation and activation and promoting extracellular matrix degradation $(11,12)$. Previous studies have shown that AKF-PD can significantly alleviate bleomycin-induced pulmonary fibrosis in mice, inhibit the expression of TGF- $\beta 1$ in bronchoalveolar lavage fluid, reduce 
the expression of type I collagen and fibronectin in lung tissue and mitigate alveolar injury (13-15). Moreover, in human lung fibroblasts stimulated with TGF- $\beta 1$, AKF-PD significantly reduces the expression levels of type I collagen and $\alpha$-SMA (16). However, the effect of AKF-PD on PQ-induced pulmonary fibrosis and its mechanism are still unclear and require further study.

$\mathrm{PI} 3 \mathrm{~K} / \mathrm{Akt} / \mathrm{mTOR}$ is a signaling pathway that plays a notable role in regulating cell proliferation, apoptosis, protein synthesis, energy metabolism and autophagy $(17,18)$. In previous years, studies have shown that the PI3K/Akt/mTOR signaling pathway is closely associated with the occurrence of pulmonary fibrosis. The activation of the PI3K/Akt/mTOR signaling pathway and insufficient autophagy promotes the occurrence and development of pulmonary fibrosis (19-21).

The present study evaluated whether AKF-PD could effectively alleviate pulmonary fibrosis caused by PQ poisoning, and analyzed the potential signaling pathways associated with its anti-fibrotic effect. First, a model of pulmonary fibrosis was established in rats to verify the effect of AKF-PD on pulmonary fibrosis induced by $\mathrm{PQ}$ poisoning. To further verify the anti-pulmonary fibrosis mechanism of AKF-PD, HPAEpiC cells were used to explore the regulation of AKF-PD on mTOR signaling pathway and autophagy. The present study provided a theoretical basis for the clinical application of AKF-PD.

\section{Materials and methods}

Reagents. PQ solution (20\%) was purchased from Syngenta Nantong Crop Protection Co., Ltd. Fluorofenidone was provided by Xiangya School of Pharmacy, Central South University. DMEM and FBS were purchased from Gibco (Thermo Fisher Scientific, Inc.). Cell Counting Kit-8 (CCK-8) and trypsin were purchased from Sigma-Aldrich (Merck KGaA). For western blot analysis, rabbit monoclonal antibodies against TGF- $\beta 1$ (1:2,000; cat. no. ab92486), $\alpha$-SMA (1:2,000; cat. no. ab32575), E-cadherin (1:1,000; cat. no. ab231303), mTOR (1:3,000; cat. no. ab134903), phosphorylated (p-)mTOR (1:3,000; cat. no. ab109268), PI3K (1:1,000; cat. no. ab32089), p-PI3K (1:1,000; cat. no. ab139317), LC3 (1:4,000; cat. no. ab232940) and p62 (1:2,000; cat. no. ab56416) were purchased from Abcam. Mouse monoclonal antibodies against $\beta$-actin $(1: 2,000$; cat. no. GB11001) were purchased from Wuhan Servicebio Technology Co., Ltd. The following ELISA kits were used: Superoxide dismutase (cat. no. SEKH-0029; Beijing Solarbio Science \& Technology Co., Ltd.), malondialdehyde (cat. no. SEKH-0053; Beijing Solarbio Science \& Technology Co., Ltd.), hydroxyproline (cat. no. SEKH-0025; Beijing Solarbio Science \& Technology Co., Ltd.), lactate dehydrogenase (cat. no. SEKH-1036; Beijing Solarbio Science \& Technology Co., Ltd.), IL-1b (cat. no. SEKH-0002; Beijing Solarbio Science \& Technology Co., Ltd.), IL-10 (cat. no. SEKH-0013; Beijing Solarbio Science \& Technology Co., Ltd.), and TNF- $\alpha$ (cat. no. SEKH-0047; Beijing Solarbio Science \& Technology Co., Ltd.).

Animals. Sprague-Dawley rats (weight 200-220 g; Four-week-old male adults), were purchased from Hunan Silaike Jingda Experimental Animal Co., Ltd. The rats were placed in a specific pathogen-free level feeding environment for routine feeding and were provided with food and water ad libitum. The incubator temperature was maintained at $22 \pm 2{ }^{\circ} \mathrm{C}$ with $40-60 \%$ humidity, on a 12 -h light/dark cycle. They were randomly divided into control, PQ, PQ + AKF-PD and AKF-PD groups. The control group received $1 \mathrm{ml} /$ day of normal saline by gavage. The PQ group received $40 \mathrm{mg} / \mathrm{kg}$ of PQ by one-time intragastric administration, then $1 \mathrm{ml} /$ day of normal saline. In the $\mathrm{PQ}+\mathrm{AKF}-\mathrm{PD}$ group, a single intragastric dose of $40 \mathrm{mg} / \mathrm{kg}$ of PQ was administered, followed by intragastric administration of $500 \mathrm{mg} / \mathrm{kg} /$ day of AKF-PD. In the AKF-PD group, $500 \mathrm{mg} / \mathrm{kg}$ of AKF-PD was intragastrically administered daily without PQ treatment. After 14 days of continuous administration, each rat was anesthetized with $1 \%$ pentobarbital sodium $(40 \mathrm{mg} / \mathrm{kg}$ ) by intraperitoneal injection before being sacrificed by decapitation. The animal death was confirmed by lack of responsiveness after $5 \mathrm{~min}$, after which the lung tissues were dissected. This study was approved by The Committee of Experimental Animals of the First People's Hospital of Hunan Province (approval no. HP2019010793).

Histopathology. The lung tissues were fixed at room temperature for $24 \mathrm{~h}$ in $4 \%$ paraformaldehyde, and dehydrated in ethanol of $70 \%$ ( $2 \mathrm{~h}$ ), $80 \%$ (overnight), 90\% (2 h) and $100 \%$ (twice in an hour) at room temperature. Following paraffin embedding, the tissues were cut into $4 \mu \mathrm{m}$ sections. The sections were stained using the hematoxylin and eosin staining kit (cat. no. C0109; Beyotime Institute of Biotechnology) and Masson' s trichrome staining kit (cat. no. C0215; Beyotime Institute of Biotechnology) according to the manufacturer's instructions. After the sections were observed and images were captured under a microscope (IX73; Olympus Corporation; magnification, $\mathrm{x} 200$ ), the degree of alveolitis and pulmonary fibrosis was evaluated according to the method described by Szapiel et al (22).

Determination of oxidative stress parameters and collagen content. The lung tissues were collected from each group, weighed, washed with $10 \%$ normal saline and subsequently centrifuged at $4^{\circ} \mathrm{C}, 10,000 \times \mathrm{g}$ for $10 \mathrm{~min}$ with an automatic biochemical analyzer. To analyze the level of oxidative stress and the degree of fibrosis in lung tissue, the amounts of SOD, MDA, and HYP was quantified according to the instructions of the ELISA kits.

Cell culture. Human alveolar epithelial cells (HPAEpiC cells) were purchased from Shanghai Tong Pai Biotechnology Co., Ltd (https://www.biomart.cn/47979/index.htm). HPAEpiC cells were cultured in DMEM containing 10\% FBS, $100 \mathrm{U} / \mathrm{ml}$ penicillin and $100 \mathrm{U} / \mathrm{ml}$ streptomycin. The cells were cultured in $37^{\circ} \mathrm{C}$ incubators under a $5 \% \mathrm{CO}_{2}$ atmosphere and the medium was changed every other day. When the cells grew to $\sim 70 \%$ confluence, HPAEpiC cells were digested with $0.25 \%$ trypsin and subcultured at a 1:3 ratio. Cells in the logarithmic growth phase were selected for experimental treatment. The cells were divided into four groups: i) Control group; ii) $\mathrm{PQ}$ group; iii) $\mathrm{PQ}+\mathrm{AKF}-\mathrm{PD}$ group; and iv) AKF-PD group. The control group cells were grown in culture medium without any intervention factors. PQ group cells were grown with the addition of $300 \mu \mathrm{mol} / 1 \mathrm{PQ}$ to stimulate cells for $24 \mathrm{~h}$. The PQ+AKF-PD group was pretreated with $1 \mathrm{mmol} / \mathrm{AKF}-\mathrm{PD}$ for 
$1 \mathrm{~h}$ and then $300 \mu \mathrm{mol} / \mathrm{l} \mathrm{PQ}$ was added for a 24-h treatment. The AKF-PD group cells were grown with the addition of only $1 \mathrm{mmol} / 1 \mathrm{AKF}-\mathrm{PD}$, without PQ. Cells were cultured in an incubator with $5 \% \mathrm{CO}_{2}$ at $37^{\circ} \mathrm{C}$.

Cell viability examination. HPAEpiC cells in the logarithmic growth phase were seeded in a 96-well plate and cultured in a $37^{\circ} \mathrm{C}$ incubator. When the cells reached $80 \%$ confluence, the cells were incubated with PQ at 0, 50, 100, 200, 300, 400, $500,600,700$ and $800 \mu \mathrm{mol} / 1$, respectively. The cell culture supernatant was collected $24 \mathrm{~h}$ later to detect the content of lactate dehydrogenase (LDH) using the ELISA kit. Then, the adherent HPAEpiC cells were cultured in a mixture of CCK-8 solution and culture medium at $37^{\circ} \mathrm{C}$ for $2 \mathrm{~h}$, and the absorbance was measured at $450 \mathrm{~nm}$ with a microplate reader. The optimal concentration of PQ was selected. Next, the cells were pretreated with increasing concentrations of AKF-PD $(0.5,1$, $1.5,2,2.5,3,3.5$ and $4 \mathrm{mmol} / \mathrm{l}$ ) for $1 \mathrm{~h}$, then stimulated with PQ for $24 \mathrm{~h}$. Cell viability was detected by CCK-8 and LDH assay.

Detection of inflammatory factors in each group of cells. The supernatant of each group was removed and inflammatory factors, including IL-1 $\beta$, IL- 6 and TNF- $\alpha$, were measured using an ELISA kit. All the operation steps were strictly in accordance with the kit instructions.

Immunofluorescence. The levels of p62 were also detected by using immunofluorescence. Coverslips covered with HPAEpiC cells were rinsed with PBS for $3 \mathrm{~min}$ at room temperature. Then, the cells were fixed with $4 \%$ paraformaldehyde for $15 \mathrm{~min}$ at room temperature and permeabilized with $0.3 \%$ Triton X-100 (Sigma-Aldrich; Merck KGaA) for $15 \mathrm{~min}$ at room temperature. The coverslips were then washed with PBS for 3 times, followed by incubating 3\% BSA (Sigma-Aldrich; Merck $\mathrm{KGaA}$ ) for $30 \mathrm{~min}$ at room temperature. The cells were incubated at $4^{\circ} \mathrm{C}$ overnight in rabbit anti-p62 primary antibody (cat. no. ab51480; 1:300; Abcam) and were then incubated in Cy3-AffiniPure Goat Anti-Rabbit IgG (cat.no. Ab45360; 1:200; Abcam) at $37^{\circ} \mathrm{C}$ for $1 \mathrm{~h}$. Finally, the cells were washed three times with PBS and then incubated with DAPI (cat. no. D9435; Sigma-Aldrich; Merck KGaA) for $5 \mathrm{~min}$ at room temperature. Then, the cells were observed and the images were captured under a fluorescence microscope. All experiments were conducted independently at least 3 times. Stained cells were visualized using an SZX12 fluorescent microscope (Olympus Corporation; magnification, x200).

Reverse transcription-quantitative PCR (RT-qPCR). Total RNA from HPAEpiC cells was extracted with TRIzol ${ }^{\circledR}$ reagent (Invitrogen; Thermo Fisher Scientific, Inc.), and reverse-transcribed to cDNA using the PrimeScript ${ }^{\mathrm{TM}}$ RT Reagent kit with gDNA Eraser (Takara Biotechnology Co., Ltd.), according to the manufacturer's protocols. The cDNA templates were amplified using the TB Green ${ }^{\circledR}$ Fast qPCR Mix (Takara Biotechnology Co., Ltd.). The following primers were used: $\alpha$-SMA, 5'-CGGGACATCAAGGAGAAACT-3' and reverse 5'-CCCATCAGGCAACTCGTAA-3'; TGF- $\beta$, forward 5'-TCGACATGGAGCTGGTGAAA-3' and reverse 5'-GAG CCTTAGTTTGGACAGATCTG-3'; $\beta$-actin, forward 5'-CAA
ATGTGTTCAGCTCAGCCAGCA-3' and reverse 5'-CTG GAAGGTGGACAGCGAGG3'. Samples were denatured at $95^{\circ} \mathrm{C}$ for $30 \mathrm{sec}$, and then PCR amplification was achieved by 40 cycles at $95^{\circ} \mathrm{C}$ for $5 \mathrm{sec}$ and $60^{\circ} \mathrm{C}$ for $15 \mathrm{sec}$ using the Applied Biosystems 7500 Real-Time PCR system (Thermo Fisher Scientific, Inc.). At least two independent experiments were conducted, and $\beta$-actin was used for normalization. The expression levels of mRNA were quantified using the $2^{-\Delta \Delta C q}$ method (23).

Western blotting analysis. The lung tissues and HPAEpiC cells were homogenized in RIPA lysis buffer (Thermo Fisher Scientific, Inc.). A protein assay kit (BCA; Thermo Scientific, Inc.) was used to measure the total protein in samples. After quantification using a BCA kit (Thermo Fisher Scientific, Inc.), a total of $10 \mu \mathrm{g}$ protein samples were separated via $10 \%$ sodium dodecyl sulfate-poly-acrylamide gel electrophoresis, and subsequently transferred to a PVDF membrane and blocked with $5 \%$ milk (w/v) at room temperature for $1 \mathrm{~h}$. The membrane was incubated overnight with primary antibodies at $4^{\circ} \mathrm{C}$. The primary antibodies used were TGF- $\beta 1$, E-cadherin, $\alpha$-SMA, Akt, p-Akt, mTOR, p-mTOR, PI3K, p-PI3K, LC3, p62 and $\beta$-actin (all Abcam). $\beta$-Actin was used as a loading control to normalize the data.

The membrane was washed 3 times with TBS containing $0.1 \%$ Tween-20 (TBST) and was incubated at room temperature for $1 \mathrm{~h}$ with horseradish peroxidase-linked anti-rabbit antibodies (cat. no. ab6822; Abcam) that were diluted 1:2,000 in TBST. An ECL kit (EMD Millipore) was used for luminescent development, a chemiluminescence instrument was used to scan the strip and Image Lab software (v 4.0; Bio-Rad) was used to analyze the gray value of the strip. Protein expression was normalized to $\beta$-actin.

Statistical analysis. GraphPad Prism (GraphPad Software, Inc.) and SPSS 22.0 (IBM Corp.) software were used for statistical analysis of the data. All experiments were repeated at least three times. The data are presented as the mean \pm standard deviation. The comparisons between two groups were analyzed using an unpaired Student's t-test. The comparisons among multiple groups were analyzed using ANOVA followed by Tukey's post hoc test. $\mathrm{P}<0.05$ was considered to indicate a statistically significant difference.

\section{Results}

AKF-PD alleviates $P Q$-induced pulmonary fibrosis in rats. To investigate the effect of AKF-PD on PQ-induced pulmonary fibrosis, HE and Masson staining were used to observe the pathological changes in the lung tissue for each group. Fig. 1A shows that in the PQ group, a significant thickening of the alveolar septa was observed with increased deposition of collagen in lung tissues at 14 days following PQ administration compared with the control (24). However, the degree of pulmonary fibrosis was significantly mitigated after 14 days of AKF-PD treatment compared with the PQ-treated rats. The degree of alveolitis and pulmonary fibrosis was graded according to Szapiel's semi-quantitative method (Fig. 1B). Compared with the control group, the degree of alveolitis and pulmonary fibrosis in the PQ group was significantly increased 

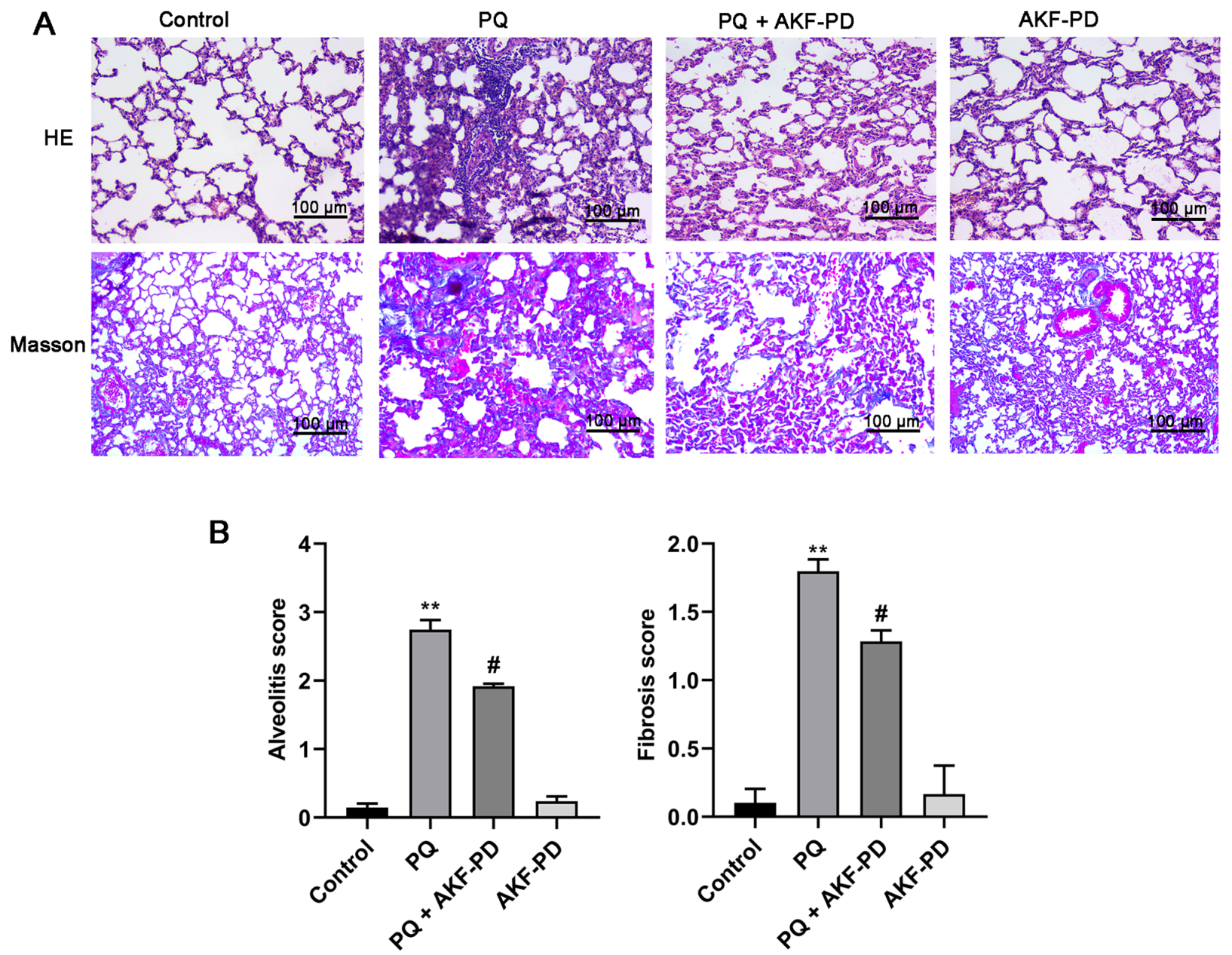

Figure 1. Fluorofenidone alleviates paraquat-induced pulmonary fibrosis in rats. (A) Morphological changes in the lung, as determined using HE staining and Masson's staining. Magnification, x200. (B) Statistical analysis of the levels of alveolitis and pulmonary fibrosis scores. " $\mathrm{P}<0.01 \mathrm{vs.}$. the control group; ${ }^{*} \mathrm{P}<0.05$ vs. the PQ group. PQ, paraquat; AKF-PD, fluorofenidone; HE, hematoxylin-eosin.
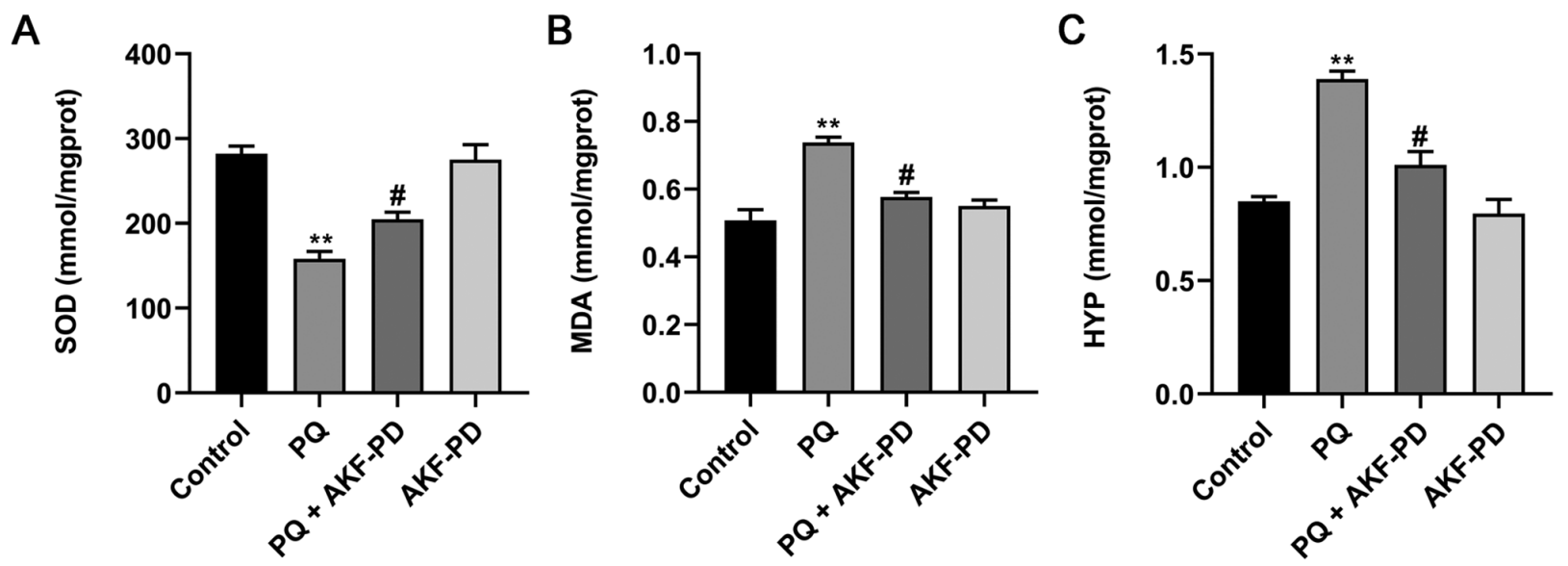

Figure 2. Effect of fluorofenidone on the oxidation parameter levels and collagen content in lung tissues. Changes in the expression of (A) SOD, (B) MDA and (C) HYP following treatment with fluorofenidone. ${ }^{* *} \mathrm{P}<0.01$ vs. the control group; ${ }^{*} \mathrm{P}<0.05$ vs. the PQ group. SOD, superoxide dismutase; MDA, malondialdehyde; HYP, hydroxyproline; PQ, paraquat; AKF-PD, fluorofenidone; mg prot, mg of protein.

$(\mathrm{P}<0.01)$. However, compared with the PQ group, the degree of alveolitis and pulmonary fibrosis in the PQ + AKF-PD group was significantly decreased $(\mathrm{P}<0.05)$. Additionally, there was no significant difference between the AKF-PD group and the control group.
$A K F-P D$ reduces oxidative stress parameters and the collagen content in lung tissue. AKF-PD and PQ were administered to the rats for 14 days. The levels of SOD, MDA and HYP in lung tissues were then evaluated to assess the impact of AKF-PD on oxidative stress and the collagen 

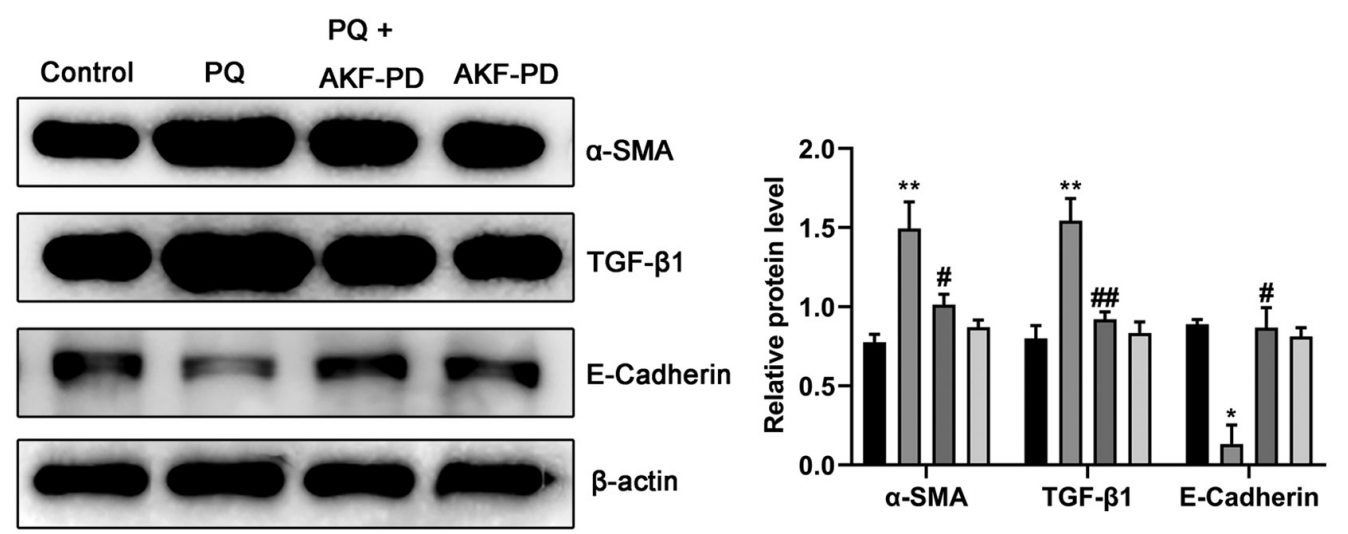
- Control
$\square P Q$
$\square P Q+A K F-P D$
$\square$ AKF-PD

Figure 3. Effect of fluorofenidone on the expression of fibrosis-related proteins in rat lung tissue. Protein levels of E-cadherin, $\alpha$-SMA and TGF- $\beta 1$ in rat lung tissue were measured by western blotting. $\beta$-actin was used as the loading control. ${ }^{*} \mathrm{P}<0.05,{ }^{* *} \mathrm{P}<0.01$ vs. the control group; ${ }^{\#} \mathrm{P}<0.05$, ${ }^{\# \#} \mathrm{P}<0.01$ vs. the $\mathrm{PQ}$ group. $\alpha$-SMA, $\alpha$-smooth muscle actin; PQ, paraquat; AKF-PD, fluorofenidone.

A

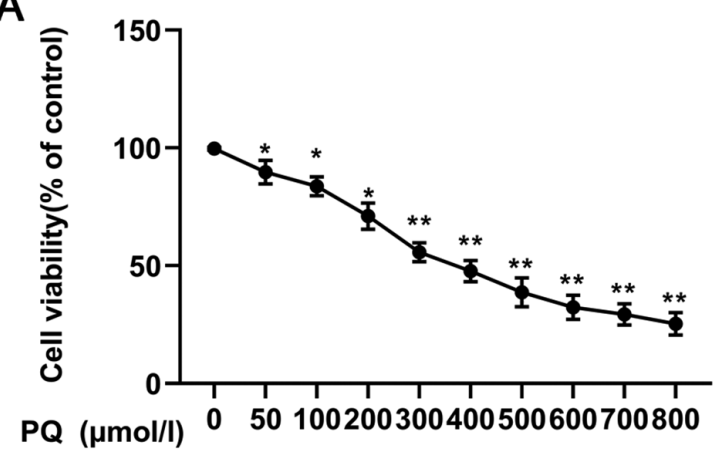

C

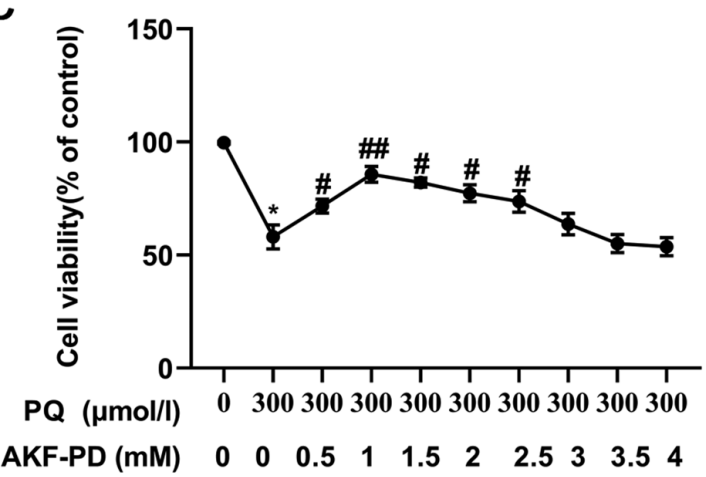

B

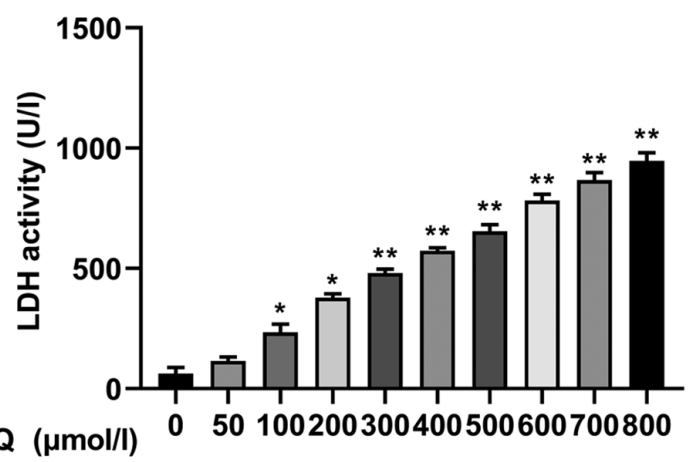

D

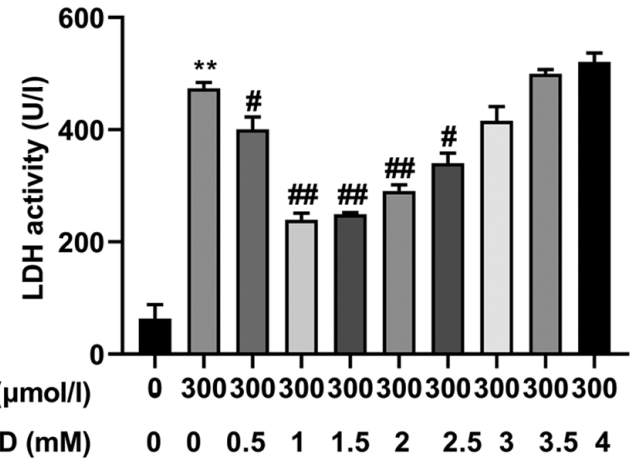

Figure 4. Effect of fluorofenidone on the viability of HPAEpiC cells. Effects of increasing concentrations of paraquat on HPAEpiC cell viability were measured using (A) CCK-8 assays and (B) LDH release assays. Effects of increasing concentrations of fluorofenidone on HPAEpiC cell viability were measured using (C) CCK-8 assays and (D) LDH release assay. ${ }^{*} \mathrm{P}<0.05,{ }^{* *} \mathrm{P}<0.01$ vs. the control group; ${ }^{*} \mathrm{P}<0.05,{ }^{\# \#} \mathrm{P}<0.01$ vs. the PQ group. CCK-8, Cell Counting Kit-8; LDH, lactate dehydrogenase; HPAEpiC, human alveolar epithelial cells; PQ, paraquat; AKF-PD, fluorofenidone.

content in PQ-poisoned rats. As shown in Fig. 2, the level of SOD significantly decreased after $14 \mathrm{~d}$ of PQ treatment, but the levels of MDA and HYP significantly increased compared with the control (all P<0.01). However, AKF-PD treatment significantly increased the level of SOD and decreased the levels of MDA and HYP compared with the PQ group (all $\mathrm{P}<0.05)$. There was no significant difference between the AKF-PD group and the control group.

Effect of AKF-PD on the expression of fibrosis-related proteins in the lung tissue of $P Q$-treated rats. To further confirm that AKF-PD can alleviate PQ-induced pulmonary fibrosis, the expression of fibrosis-related proteins was measured using western blotting in the lung tissue of rats in each group. As shown in Fig. 3 , the expression levels of $\alpha$-SMA and TGF- $\beta 1$ protein in the lung tissue of the PQ group significantly increased $(\mathrm{P}<0.01)$, whereas the expression level of $\mathrm{E}$-cadherin significantly decreased compared with the control $(\mathrm{P}<0.05)$. However, AKF-PD treatment significantly decreased the protein levels of $\alpha$-SMA $(\mathrm{P}<0.05)$ and TGF- $\beta 1(\mathrm{P}<0.01)$ and increased the level of $\mathrm{E}$-cadherin $(\mathrm{P}<0.05)$. Moreover, the expression levels of $\alpha$-SMA, TGF- $\beta 1$ and E-cadherin in the AKF-PD group were not significantly different from those in the control group. 
A

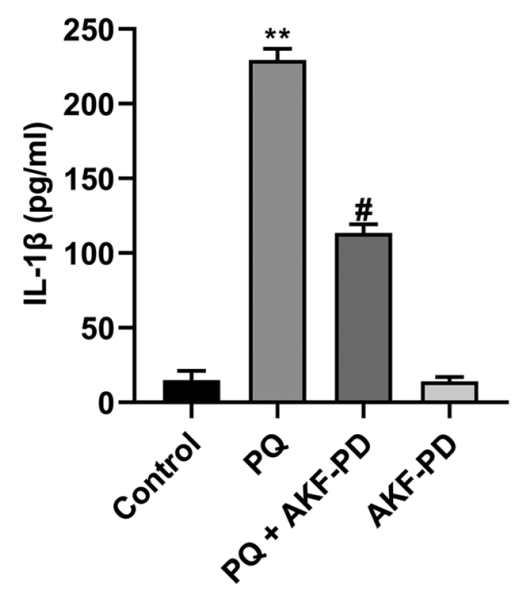

B

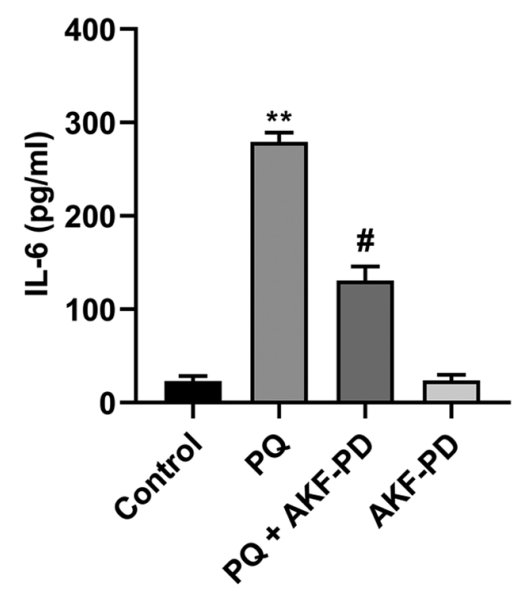

C

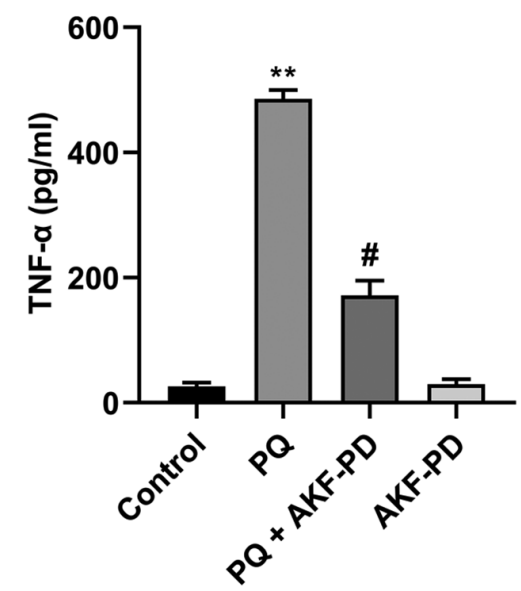

Figure 5. Effect of fluorofenidone on the changes of inflammatory factors after paraquat poisoning. Concentrations of inflammatory factors (A) IL-1 $\beta$, (B) IL-6 and (C) TNF- $\alpha$ were detected using an ELISA kit. ${ }^{* *} \mathrm{P}<0.01$ vs. the control group; ${ }^{*} \mathrm{P}<0.05$ vs. the $\mathrm{PQ}$ group. $\mathrm{PQ}$, paraquat; AKF-PD, fluorofenidone.

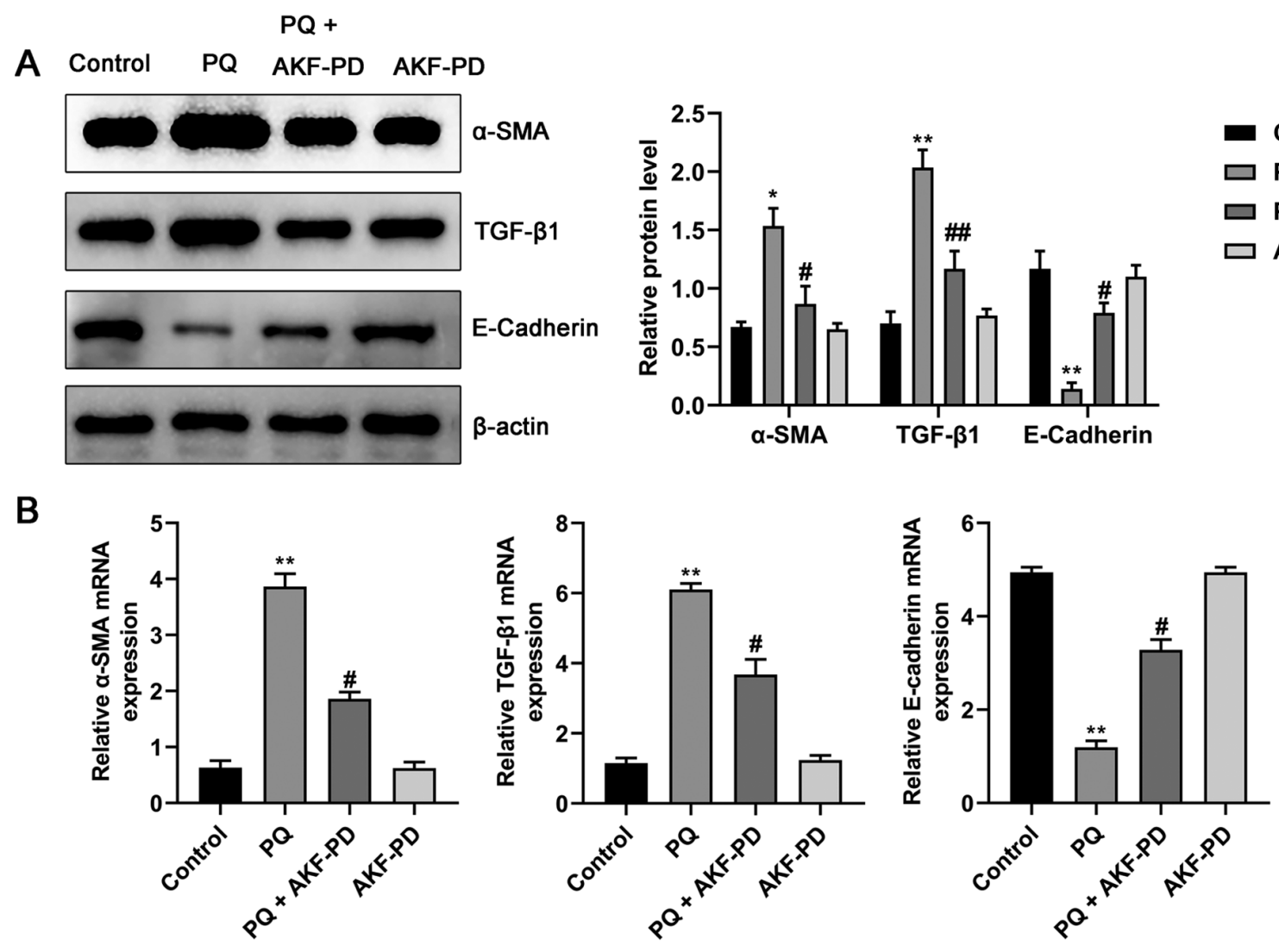

Figure 6. Effects of fluorofenidone on $\alpha$-SMA, TGF- $\beta 1$ and E-cadherin expression in HPAEpiC cells. (A) Protein level of $\alpha$-SMA, TGF- $\beta 1$ and E-cadherin in HPAEpiC cells were detected by western blotting. (B) mRNA levels of $\alpha$-SMA, TGF- $\beta 1$ and E-cadherin in HPAEpiC cells were detected by reverse transcription-quantitative PCR. ${ }^{*} \mathrm{P}<0.05,{ }^{* *} \mathrm{P}<0.01$ vs. the control group; ${ }^{\#} \mathrm{P}<0.05,{ }^{\# \#} \mathrm{P}<0.01$ vs. the PQ group. $\alpha$-SMA, $\alpha$-smooth muscle actin; HPAEpiC, human alveolar epithelial cells; PQ, paraquat; AKF-PD, fluorofenidone.

Effect of AKF-PD on cell viability stimulated by $P Q$. To determine the effect of AKF-PD on the viability of HPAEpiC cells, CCK-8 assays and LDH release assays were performed. The cells were incubated with different concentrations of $\mathrm{PQ}$, and cell viability was measured at $24 \mathrm{~h}$. As shown in Fig. 4A, cell viability gradually decreased significantly with the increase in the PQ concentration, and Fig. 4B shows how
LDH leakage gradually increased significantly (all $\mathrm{P}<0.05$ ). The median lethal dose $\left(\mathrm{IC}_{50}\right)$ of PQ was $300 \mu \mathrm{mol} / 1$ and, therefore, the concentration of $300 \mu \mathrm{mol} / 1$ was used in subsequent experiments. Next, the cells were treated with increasing concentrations of AKF-PD for $1 \mathrm{~h}$, then stimulated with $300 \mu \mathrm{mol} / \mathrm{l} \mathrm{PQ}$ for $24 \mathrm{~h}$. As shown in Fig. 4C and D, AKF-PD $(0.5,1,1.5,2$ and $2.5 \mathrm{mmol} / \mathrm{l})$ significantly ameliorated the 


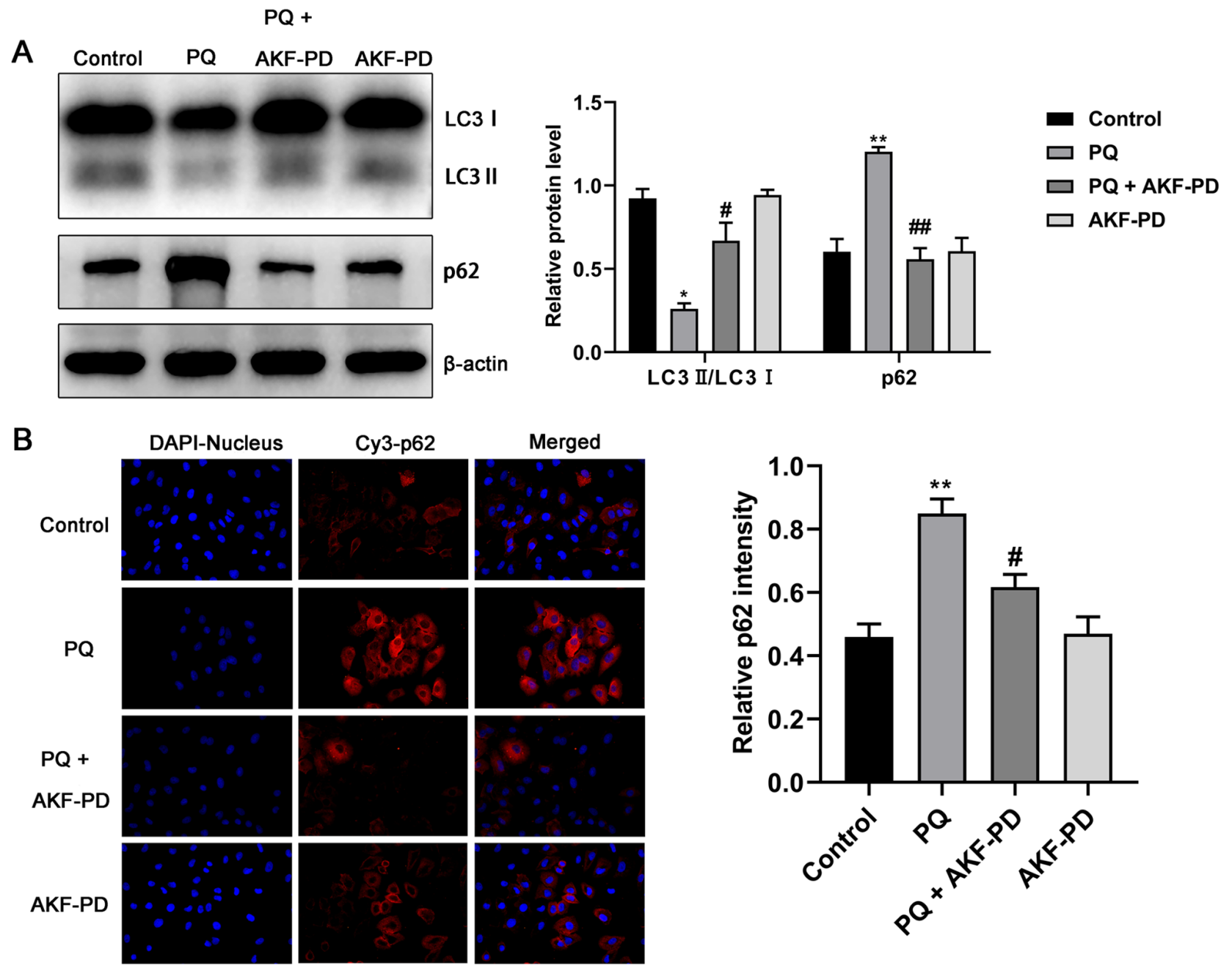

Figure 7. Fluorofenidone upregulates autophagy in paraquat-stimulated HPAEpiC cells. (A) Protein levels of LC3 and p62 in HPAEpiC cells were detected by western blotting. (B) Expression levels of p62 in HPAEpiC cells were measured via immunofluorescence staining. Magnification, $\mathrm{x} 200 .{ }^{*} \mathrm{P}<0.05,{ }^{* *} \mathrm{P}<0.01 \mathrm{vs}$. the control group; ${ }^{~} \mathrm{P}<0.05,{ }^{\# \#} \mathrm{P}<0.01$ vs. the PQ group. HPAEpiC, human alveolar epithelial cells; PQ, paraquat; AKF-PD, fluorofenidone.

decrease in HPAEpiC cell viability induced by PQ, with the optimum concentration at $1 \mathrm{mmol} / 1(\mathrm{P}<0.05)$. Therefore, the concentration of $1 \mathrm{mmol} / \mathrm{l}$ was used for subsequent experiments.

Effect of $A K F-P D$ on the expression of $P Q$-induced inflammatory factors. The concentrations of IL-1 $\beta$, IL-6 and TNF- $\alpha$ in the supernatant of HPAEpiC cells were quantitated using ELISA. As shown in Fig. 5, IL-1 $\beta$, IL-6 and TNF- $\alpha$ levels in the PQ group significantly increased compared with the control group $(\mathrm{P}<0.01)$. Compared with the $\mathrm{PQ}$ group, however, IL-1 $\beta$, IL-6 and TNF- $\alpha$ levels in the PQ + AKF-PD group significantly decreased $(\mathrm{P}<0.05)$. These results suggested that AKF-PD could inhibit PQ-induced inflammation in HPAEpiC cells.

Effect of AKF-PD on the $\alpha$-SMA, TGF- $\beta 1$ and E-cadherin expression in HPAEpiC cells. To further verify the effect of AKF-PD on PQ-induced pulmonary fibrosis and its potential mechanism, the protein and mRNA levels of $\alpha$-SMA, TGF- $\beta 1$ and E-cadherin in HPAEpiC cells were measured using western blotting and RT-qPCR. As shown in Fig. 6A and B, compared with the control group, the mRNA and protein levels of $\alpha$-SMA and TGF- $\beta 1$ were significantly increased, and the expression of E-cadherin protein was significantly decreased (all $\mathrm{P}<0.05)$. However, compared with the $\mathrm{PQ}$ group, the expression levels of $\alpha$-SMA and TGF- $\beta 1$ in the PQ+AKF-PD group were significantly decreased, while the expression of E-cadherin was significantly increased $(\mathrm{P}<0.05)$.

AKF-PD inhibits the development of pulmonary fibrosis by increasing autophagy. The expression levels of autophagy-related proteins LC3 and p62 were measured using western blotting and an immunofluorescence test, and the results are shown in Fig. 7A. In the PQ group, LC3 II/LC3 I was significantly decreased $(\mathrm{P}<0.05)$ and p62 increased $(\mathrm{P}<0.01)$ compared with the control. However, in the PQ + AKF-PD group, LC3 II/LC3 I expression significantly increased $(\mathrm{P}<0.05)$ while p62 decreased $(\mathrm{P}<0.01)$ compared with the PQ group. Moreover, the immunofluorescence images in Fig. 7B were consistent with the aforementioned results, which indicated that AKF-PD pretreatment significantly decreased the expression of p62 compared with PQ treated cells.

AKF-PD inhibits the development of pulmonary fibrosis by regulating the $P I 3 K / A k t / m T O R$ signaling pathway. 
$\mathbf{P Q}+$
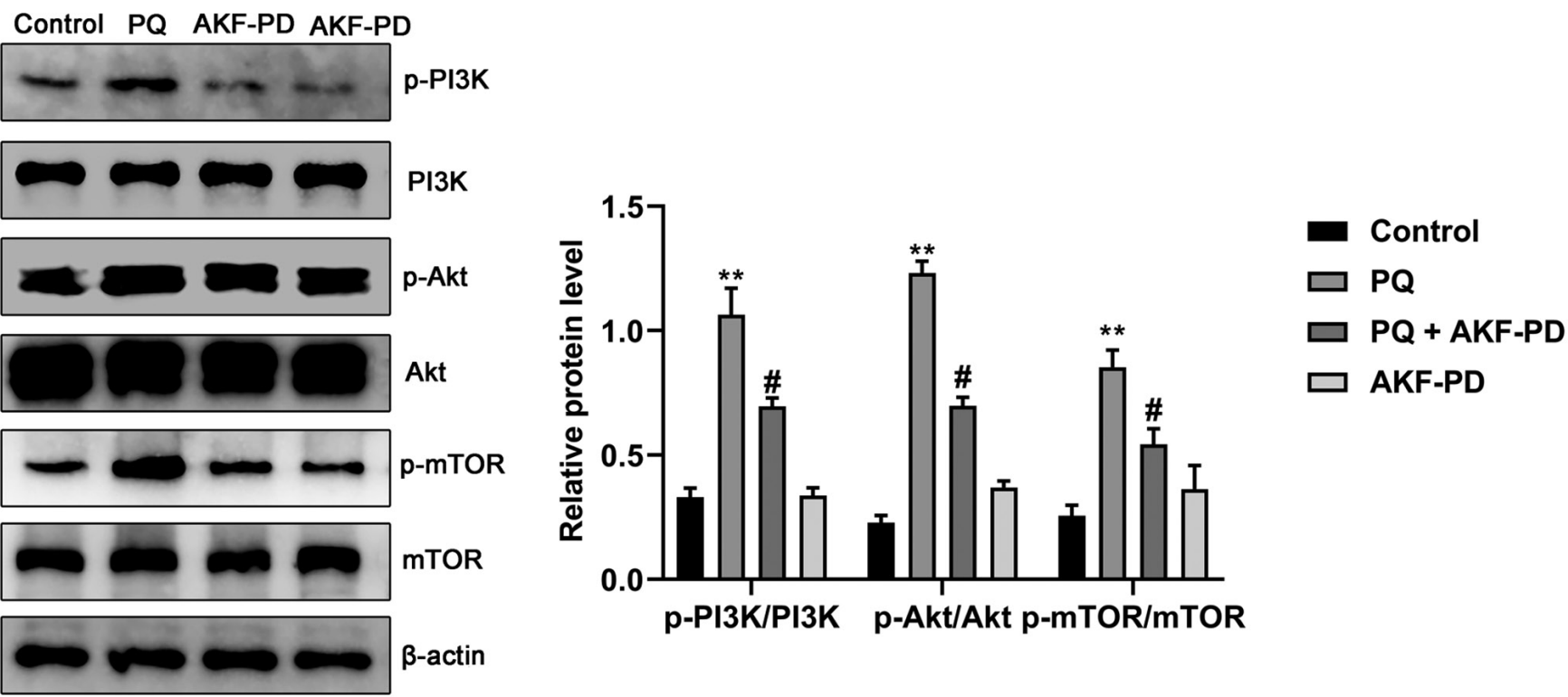

Figure 8. Effects of fluorofenidone on the expression of PI3K/Akt/mTOR signaling pathway-related proteins in lung epithelial cells. The protein levels of p-Akt, Akt, p-mTOR, mTOR, p-PI3K and PI3K were measured by western blotting. $\beta$-actin was employed as the loading control. ${ }^{* *} \mathrm{P}<0.01$ vs. the control group; ${ }^{\#} \mathrm{P}<0.05$ vs. the PQ group. PQ, paraquat; AKF-PD, fluorofenidone; $\mathrm{p}-$-, phosphorylated.

To investigate the potential underlying mechanisms of AKF-PD, the expression levels of proteins associated with the PI3K/Akt/mTOR signaling pathway were observed. As seen in Fig. 8, the p-Akt/Akt, p-mTOR/mTOR and $\mathrm{p}-\mathrm{PI} 3 \mathrm{~K} / \mathrm{PI} 3 \mathrm{~K}$ ratios in the PQ group were significantly upregulated compared with the control group (all $\mathrm{P}<0.01$ ), indicating that the PI3K/Akt/mTOR signaling pathway was activated during PQ-induced pulmonary fibrosis. However, AKF-PD treatment significantly reversed the $\mathrm{PQ}$-induced increase in p-Akt/Akt, p-mTOR/mTOR and p-PI3K/PI3K expression (all $\mathrm{P}<0.05$ ). Furthermore, there was no significant difference in the protein expression levels of p-Akt/Akt, p-mTOR/mTOR or $\mathrm{p}-\mathrm{PI} 3 \mathrm{~K} / \mathrm{PI} 3 \mathrm{~K}$ protein in the cells treated with only AKF-PD compared with the control group.

\section{Discussion}

PQ primarily accumulates in lung tissue, where it causes alveolar epithelial cell rupture, edema, interstitial changes and inflammatory cell infiltration. Moreover, increased fibroblast proliferation and collagen deposition eventually lead to pulmonary fibrosis (25). Therefore, intervention and mitigation of pulmonary fibrosis are potential strategies for treating PQ poisoning.

The results of HE and Masson staining in the present study showed that AKF-PD treatment significantly reduced the degree of alveolitis and fibrosis. It was preliminarily confirmed that AKF-PD reduced the pathological damage to lung tissue in PQ-poisoned rats. In addition, after PQ poisoning, the body releases a large amount of oxygen free radicals, leading to a large consumption of SOD that results in an increase in MDA (26). These two oxidative stress markers reflect the level of damage caused by oxygen-free radicals (27). HYP is an indicator used to evaluate fibroblast proliferation and collagen deposition (28). Therefore, the present study evaluated the toxicity of PQ by measuring the concentrations of
MDA, SOD and HYP in lung tissue. The results indicated that AKF-PD reduced the MDA and HYP levels in the lung tissue of rats with $\mathrm{PQ}$ poisoning and increased SOD, indicating that AKF-PD can effectively alleviate PQ-induced oxidative stress and collagen deposition.

Epithelial-mesenchymal transition (EMT) is also an notable part in the pathological process of pulmonary fibrosis. Alveolar epithelial cells undergo continuous damage and gradually transform into mesenchymal cells (29). At the same time, the expression of E-cadherin decreases and the expression of $\alpha$-SMA increases (30). TGF- $\beta 1$ is a key regulator of EMT, and excessive activation of TGF- $\beta 1$ can induce EMT in alveolar epithelial cells, which promotes the occurrence and development of pulmonary fibrosis (31).

In the present study, western blotting results showed that AKF-PD reduced the expression of TGF- $\beta 1$ and $\alpha$-SMA in the lung tissue of $\mathrm{PQ}$-poisoned rats, and increased the expression of E-cadherin. These results indicated that AKF-PD may reduce the content of fibrin by inhibiting the expression of TGF- $\beta 1$, thereby alleviating pulmonary fibrosis. Furthermore, HPAEpiC cells were used to determine the specific function of AKF-PD during the pathogenesis of EMT. In addition, the current study demonstrated that AKF-PD $(0.5,1,1.5,2$ and $2.5 \mathrm{mmol} / \mathrm{l})$ pretreatment significantly ameliorated the decrease in HPAEpiC cell viability induced by PQ. Notably, the protective ability of AKF-PD decreased after $1 \mathrm{mmol} / \mathrm{l}$, suggesting $1 \mathrm{mmol} / \mathrm{l}$ may be the maximum safety limit of AKF-PD, and excessive drug concentration can produce toxic effects on cells.

Autophagy is generally considered to be a lysosomal-dependent self-degradation pathway in the development of eukaryotes that plays a notable role in maintaining cell homeostasis (32). A decrease in autophagy activity is considered to be the key process in the pathogenesis of pulmonary fibrosis (33). According to previous reports, insufficient autophagy promotes the expression of $\alpha$-SMA and fibronectin 
and increases the deposition of the extracellular matrix (34-36). LC3 and p62 are markers of autophagy. When the expression level of LC3 II/LC3 I decreases, a large amount of p62 accumulates in cells, which then reduces autophagy activity and promotes lung fibrosis (37). The present study demonstrated that AKF-PD treatment decreased the expression of TGF- $\beta 1$ and $\alpha$-SMA and increased autophagy, indicating that AKF-PD can inhibit the EMT by enhancing autophagy, thereby alleviating pulmonary fibrosis.

The PI3K/Akt/mTOR signaling pathway plays a key regulatory role in autophagy (38). Under physiological conditions, growth factors and cytokines activate tuberous sclerosis 1 proteins 1 and 2 by activating PI3K and phosphorylating Akt, which then activates mTOR. Activated mTOR (p-mTOR) promotes protein synthesis and inhibits autophagy by phosphorylating downstream ribosomal protein S6 kinase 1 and eukaryotic translation initiation factor $4 \mathrm{E}$ binding protein 1 (39). Continuous activation of the PI3K/Akt/mTOR signaling pathway has been previously observed in lung fibroblasts of patients with pulmonary fibrosis; wherein the expression of p62 increased and the expression of LC3 II/LC3 I decreased (40). This showed that the activation of the PI3K/Akt/mTOR pathway in alveolar epithelial cells decreases autophagy activity and subsequently contributes to the pathogenesis of pulmonary fibrosis. In the present study, AKF-PD led to a decrease in p-Akt/Akt, p-mTOR/mTOR and p-PI3K/PI3K ratios, demonstrating that AKF-PD can inhibit the activation of the PI3K/Akt/mTOR signaling pathway induced by PQ.

In conclusion, the current study indicated that AKF-PD inhibited the activation of the PI3K/Akt/mTOR signaling pathway, enhanced autophagy and suppressed the occurrence of EMT, thereby alleviating the pulmonary fibrosis caused by PQ poisoning. The present study explored the effect and mechanism of AKF-PD on pulmonary fibrosis caused by PQ poisoning and laid the groundwork for investigation into future therapeutic strategies that can be used to treat pulmonary fibrosis.

\section{Acknowledgements}

Not applicable.

\section{Funding}

This work was supported by The Scientific Research Project of Hunan Provincial Commission of Health and Family Planning (grant no. B2017076), The Changsha Science and Technology Department (grant no. kq1901051).

\section{Availability of data and materials}

The datasets used and/or analyzed during the current study are available from the corresponding author on reasonable request.

\section{Authors' contributions}

YJ and WL conceived the study. FJ and SL designed the methodology, wrote the original draft manuscript, and revised and edited the manuscript. XY, TW, and ZC analyzed the data. WL and YJ confirm the authenticity of all the raw data. All authors have read and approved the final manuscript.

\section{Ethics approval and consent to participate}

The experimental protocol used in this study was approved by The Committee of Experimental Animals of the First People's Hospital of Hunan Province.

\section{Patient consent for publication}

Not applicable.

\section{Competing interests}

The authors declare that they have no competing interests.

\section{References}

1. Samsamshariat S, Vedaei A, Jahangiri S, Gavarti MB, Sami R, Taheri A and Dorooshi G: Report of a case of paraquat poisoning and mediastinal involvement. Adv Biomed Res 10: 5, 2021.

2. Zhu Y, Tan J, Xie H, Wang J, Meng X and Wang R: HIF-1a regulates EMT via the Snail and $\beta$-acatenin pathways in paraquat poisoning-induced early pulmonary fibrosis. J Cell Mol Med 20: 688-697, 2016.

3. Suntres ZE: Role of antioxidants in paraquat toxicity. Toxicology 180: 65-77, 2002.

4. Xiao Q, Wang W, Qi H, Gao X, Zhu B, Li J and Wang P: Continuous hemoperfusion relieves pulmonary fibrosis in patients with acute mild and moderate paraquat poisoning. J Toxicol Sci 45: 611-617, 2020.

5. Tao W, Shu Y, Miao Q and Zhu Y: Attenuation of hyperoxia-induced lung injury in rats by adrenomedullin. Inflammation 35: 150-157, 2012.

6. Shen $\mathrm{H}$, Wu N, Wang Y, Zhao H, Zhang L, Li T and Zhao M: Chloroquine attenuates paraquat-induced lung injury in mice by altering inflammation, oxidative stress and fibrosis. Int Immunopharmacol 46: 16-22, 2017.

7. Bai L, Li A, Gong C, Ning X and Wang Z: Protective effect of rutin against bleomycin induced lung fibrosis: Involvement of TGF- $\beta 1 /$ $\alpha$-SMA/Col I and III pathway. Biofactors 46: 634-644, 2020.

8. Qian J, Ye Y, Lv L, Zhu C and Ye S: FTY720 attenuates paraquat-induced lung injury in mice. Int Immunopharmacol 21: 426-431, 2014.

9. Xu S, Hu H, Jiang Z, Tang S, Zhou Y, Sheng J, Chen J and Cao Y: APACHE score, severity index of paraquat poisoning, and serum lactic acid concentration in the prognosis of paraquat poisoning of Chinese patients. Pediatr Emerg Care 31: 117-121, 2015.

10. Yang H, Zhang W, Xie T, Wang X and Ning W: Fluorofenidone inhibits apoptosis of renal tubular epithelial cells in rats with renal interstitial fibrosis. Braz J Med Biol Res 52: e8772, 2019.

11. Song C, He L, Zhang J, Ma H, Yuan X, Hu G, Tao L, Zhang J and Meng J: Fluorofenidone attenuates pulmonary inflammation and fibrosis via inhibiting the activation of NALP3 inflammasome

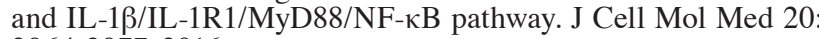
2064-2077, 2016.

12. Ma H, Peng Z, Hu G and Tao L: Effect and mechanism of fluorofenidone on organ fibrosis. Zhong Nan Da Xue Xue Bao Yi Xue Ban 40: 208-213, 2015 (In Chinese).

13. Meng J, Zou Y, Hu C, Zhu Y, Peng Z, Hu G, Wang Z and Tao L: Fluorofenidone attenuates bleomycin-induced pulmonary inflammation and fibrosis in mice via restoring caveolin 1 expression and inhibiting mitogen-activated protein kinase signaling pathway. Shock 38: 567-573, 2012.

14. Tang J, Li J, Li G, Zhang H, Wang L, Li D and Ding J: Spermidine-mediated poly(lactic-co-glycolic acid) nanoparticles containing fluorofenidone for the treatment of idiopathic pulmonary fibrosis. Int J Nanomedicine 12: 6687-6704, 2017.

15. Xu M, Li S, Wang J, Huang S, Zhang A, Zhang Y, Gu W, Yu X and Jia Z: Cilomilast ameliorates renal tubulointerstitial fibrosis by inhibiting the TGF- $\beta 1-$ Smad2/3 signaling pathway. Front Med (Lausanne) 7: 626140, 2021

16. Liu J, Song C, Xiao Q, Hu G, Tao L and Meng J: Fluorofenidone attenuates TGF- $\beta 1$-induced lung fibroblast activation via restoring the expression of caveolin-1. Shock 43: 201-207, 2015. 
17. Saxton RA and Sabatini DM: mTOR signaling in growth metabolism, and disease. Cell 168: 960-976, 2017.

18. Alayev A and Holz MK: mTOR signaling for biological control and cancer. J Cell Physiol 228: 1658-1664, 2013.

19. Gui YS, Wang L, Tian X, Xue L, Ma A, Zhou W, Zeng N, Ji Z Cai $\mathrm{B}$, Zhang $\mathrm{H}$, et al: $\mathrm{mTOR}$ overactivation and compromised autophagy in the pathogenesis of pulmonary fibrosis. PLoS One 10: e0138625, 2015.

20. Gui X, Chen H, Cai H, Sun L and Gu L: Leptin promotes pulmonary fibrosis development by inhibiting autophagy via PI3K/Akt/mTOR pathway. Biochem Biophys Res Commun 498: 660-666, 2018

21. Rieg AD, Said S, Carolin A, Eva V, Rolf R, Stefan U and Christian M: PDGF-BB regulates the pulmonary vascular tone: Impact of prostaglandins, calcium, MAPK- and $\mathrm{PI} 3 \mathrm{~K} / \mathrm{AKT} / \mathrm{mTOR}$ signalling and actin polymerisation in pulmonary veins of guinea pigs. Respir Res 19: 120, 2018.

22. Szapiel SV, Elson NA, Fulmer JD, Hunninghake GW and Crystal RG: Bleomycin-induced interstitial pulmonary disease in the nude, athymic mouse. Am Rev Respir Dis 120: 893-899, 1979.

23. Livak KJ and Schmittgen TD: Analysis of relative gene expression data using real-time quantitative PCR and the 2(-Delta Delta C(T)) method. Methods 25: 402-408, 2001

24. Liu MW, Su MX, Tang DY, Hao L, Xun XH and Huang YQ: Ligustrazin increases lung cell autophagy and ameliorates paraquat-induced pulmonary fibrosis by inhibiting PI3K/Akt/mTOR and hedgehog signalling via increasing miR-193a expression. BMC Pulm Med 19: 35, 2019.

25. Raghu G, Rochwerg B, Zhang Y, Garcia CA, Azuma A, Behr J, Brozek JL, Collard HR, Cunningham W, Homma S, et al: An official ATS/ERS/JRS/ALAT clinical practice guideline: Treatment of idiopathic pulmonary fibrosis. An update of the 2011 clinical practice guideline. Am J Respir Crit Care Med 192: e3-19, 2015.

26. Wang J, Qiao L, Li S and Yang G: Protective effect of ginsenoside $\mathrm{Rb} 1$ against lung injury induced by intestinal ischemia-reperfusion in rats. Molecules 18: 1214-1226, 2013.

27. Wu J, Chu Z, Ruan Z, Wang X, Dai T and Hu X: Changes of intracellular porphyrin, reactive oxygen species, and fatty acids profiles during inactivation of methicillin-resistant staphylococcus aureus by antimicrobial blue light. Front Physiol 9: 1658, 2018.

28. Yamada M, Kuwano K, Maeyama T, Hamada N Michihiro Yoshimi, Yoichi Nakanishi, and Kasper M: Dual-immunohistochemistry provides little evidence for epithelial-mesenchymal transition in pulmonary fibrosis. Histochem Cell Biol 129: 453-462, 2008.
29. Molina-Molina M, Machahua-Huamani C, Vicens-Zygmunt V, Llatjós R, Escobar I, Sala-Llinas E, Luburich-Hernaiz P, Dorca J and Montes-Worboys A: Anti-fibrotic effects of pirfenidone and rapamycin in primary IPF fibroblasts and human alveolar epithelial cells. BMC Pulm Med 18: 63, 2018.

30. Chitra P, Saiprasad G, Manikandan R and Sudhandiran G: Berberine inhibits Smad and non-Smad signaling cascades and enhances autophagy against pulmonary fibrosis. J Mol Med (Berl) 93: 1015-1031, 2015.

31. Liu Z, Li Y, Song H, He J, Li G, Zheng Y and Li B: Collagen peptides promote photoaging skin cell repair by activating the TGF- $\beta /$ Smad pathway and depressing collagen degradation. Food Funct 10: 6121-6134, 2019.

32. Eskelinen E: Autophagy: Supporting cellular and organismal homeostasis by self-eating. Int J Biochem Cell Biol 111: 1-10, 2019

33. Lv X, Li K and Hu Z: Autophagy and Pulmonary Fibrosis. Adv Exp Med Biol: 1207: 569-579, 2020.

34. Tian K, Chen P, Liu Z, Si S, Zhang Q, Mou Y, Han L, Wang Q and Zhou X: Sirtuin 6 inhibits epithelial to mesenchymal transition during idiopathic pulmonary fibrosis via inactivating TGF-31/Smad3 signaling. Oncotarget 8: 61011-61024, 2017.

35. Mu E, Wang J, Chen L, Lin S, Chen J and Huang S: Ketogenic diet induces autophagy to alleviate bleomycin-induced pulmonary fibrosis in murine models. Exp Lung Res 47: 26-36, 2021.

36. Im J, Hergert P and Nho R: Reduced FoxO3a expression causes low autophagy in idiopathic pulmonary fibrosis fibroblasts on collagen matrices. Am J Physiol Lung Cell Mol Physiol 309: L552-L561, 2015.

37. Xie T, Xu Q, Wan H, Xing S, Shang C, Gao Y and He Z: Lipopolysaccharide promotes lung fibroblast proliferation through autophagy inhibition via activation of the PI3K-Akt-mTOR pathway. Lab Invest 99: 625-633, 2019.

38. Patel AS, Lin L, Geyer A, Haspel JA, An CH, Cao J, Rosas IO and Morse D: Autophagy in idiopathic pulmonary fibrosis. PLoS One 3: e41394, 2012.

39. Jung C, Ro S, Cao J, Otto $\mathrm{N}$ and Kim D: mTOR regulation of autophagy. FEBS Lett 584: 1287-1295, 2010.

40. Nho R and Hergert P: IPF fibroblasts are desensitized to type I collagen matrix-induced cell death by suppressing low autophagy via aberrant Akt/mTOR kinases. PLoS One 9: e94616, 2014.

This work is licensed under a Creative Commons Attribution-NonCommercial-NoDerivatives 4.0 International (CC BY-NC-ND 4.0) License. 